\title{
THE EXTREMUM LAW
}

\author{
W. H. GOTTSCHALK
}

The extremum law is defined to be the class $E$ of all statements $S$ such that $S$ is equivalent to the axiom of choice and such that the conclusion of $S$ asserts the existence of an extremal (maximal or minimal) element. A member of $E$ is called a form of the extremum law. Various forms of the extremum law are known under the names of Zorn's Lemma, Hausdorff maximality principle, etc.

The purpose of this note is to point out the following forms of the extremum law, of which (1) is particularly simple:

(1) If $\alpha$ is a binary relation, then there exists a maximal set $A$ such that $A \times A \subset \alpha$.

(2-5) If $X$ is a set provided with a binary relation, then there exists a maximal \{coherent $\}$ asymmetric \{chained \{antisymmetric subset $A$ of $X$. [Form (2) was pointed out by A. D. Wallace, $A$ substitute for the axiom of choice, Bull. Amer. Math. Soc. vol. 50 (1944) p. 278.]

(6) If $I$ is a nonvacuous finite set and if $\alpha$ is a set of functions on $I$, then there exists a maximal set $A$ such that $A^{I} \subset \alpha$.

With respect to a binary relation $\alpha$ in a set $X$, a subset $A$ of $X$ is \{coherent \{asymmetric\} \{chained \{antisymmetric if and only if $\{A \times A \subset \alpha \cup \tilde{\alpha}\} \quad\left\{A \times A \subset \alpha^{\prime} \cup \tilde{\alpha}^{\prime}\right\} \quad\{A \times A \subset \alpha \cup \tilde{\alpha} \cup \Delta\} \quad\{A \times A$ $\left.\subset \alpha^{\prime} \cup \tilde{\alpha}^{\prime} \cup \Delta\right\}$ where $\alpha^{\prime}$ is the complement of $\alpha$ in $X \times X, \tilde{\alpha}$ is the converse of $\alpha$, and $\Delta$ is the identity relation in $X$. Forms $\{(2)$ and (3) $\}$ $\{(4)$ and (5) $\}$ are complement dual, that is, to pass from one to the other replace $\alpha$ by $\alpha^{\prime}$. The pairwise equivalence of (1)-(6) and familiar forms of the extremum law is clear.

Form (6) is the extension of (1) from binary to finitary relations. If the word "finite" is deleted from (6), then the resulting statement is false. This is shown by the following example: $I$ is the set of all positive integers, $A_{n}(n \in I)$ is the set of the first $n$ positive integers, and $\alpha=\bigcup_{n \in I} A_{n}^{I}$.

It is clear that (1)-(6) may be strengthened by requiring that the maximal set $A$ contain a given set $A_{0}$ with the relevant property.

University of Pennsylvania

Presented to the Society, February 23, 1952; received by the editors December 26, 1951. 